\title{
A Case Report of Tetanus Patient Showing Trismus and Dysphagia as Early Symptoms
}

\author{
Seo-Hee Jang, Jin-Seok Byun, Jae-Kwang Jung, Jae-Kap Choi \\ Department of Oral Medicine, School of Dentistry, Kyungpook National University, Daegu, Korea
}

Received September 7, 2020

Revised September 10, 2020

Accepted September 10, 2020

\section{Correspondence to:}

Jae-Kap Choi

Department of Oral Medicine, School of

Dentistry, Kyungpook National University,

2175 Dalgubeol-daero, Jung-gu, Daegu

41940, Korea

Tel: $+82-53-600-7321$

Fax: +82-53-426-2195

E-mail: jhchoi@knu.ac.kr

https://orcid.org/0000-0001-6773-7507
Tetanus is a fatal disease caused by the infection Clostridium tetani found in animal feces and soil. It is a vaccine-preventable disease and rarely occurs in developed countries. However, approximately 30 cases still occur annually in South Korea. Tetanus, commonly called lockjaw, cause contraction of the masseter muscles in the early stage, resulting in trismus as the first symptom. As it progresses, spasm extends to various muscles in the face, neck, shoulder, and back, leading to distorted facial expression, dysphagia, backward arching of the body, dyspnea, and even death. Early diagnosis of tetanus is critical because it can quickly become fatal if left untreated. We present a case of trismus caused by tetanus and emphasize the importance of early diagnosis of acute trismus.

Key Words: Dysphagia; Tetanus; Trismus

\section{INTRODUCTION}

Tetanus is a life-threatening disease caused by the infection Clostridium tetani, a gram-positive anaerobic bacterium frequently found in animal feces and soil [1]. The neurotoxin (tetanospasmin) produced by $C$. tetani causes painful muscle spasms and muscle contraction by inhibiting the release of $\gamma$-aminobutyric acid (GABA) and glycine [2]. Tetanus is also commonly called lockjaw, reflecting the frequent involvement of pathologic spasm in the masseter muscles as the first symptom in most cases (50\%-70\%) [3]. As it progresses, spasm extends to various muscles in the face, neck, shoulder, and back, leading to distorted facial expression, dysphagia, backward arching of the body, dyspnea, and even death [4]. While this dangerous disease rarely occurs in developed countries due to effective immunization programs, an estimated of over 50,000 deaths caused by tetanus was still reported worldwide in 2015 [5].
Early diagnosis and prompt treatment of tetanus are critical because it can quickly become fatal if left untreated. This report describes a patient with tetanus who presented with trismus and dysphagia as the early symptoms of the disease.

\section{CASE REPORT}

A 48-year-old female patient visited the Department of Oral Medicine, Kyungpook National University Dental Hospital, with the chief complaints of trismus and dysphagia. The symptoms occurred 5 days ago without apparent initiating events, and it progressively worsened over time. She had not taken any new medication or had surgery, including dental treatment, before symptoms onset. She had no history of head and neck trauma but she fell to the ground and obtained knee abrasion about 3 weeks ago and her legs were hurt by a rusty nail 1 week ago. She visited 
the emergency room of a hospital due to acute difficulty of opening the jaw 3 days before coming to our department and was diagnosed with mandibular left third molar pericoronitis. She was prescribed antibiotics for 3 days, however, the opening difficulty did not improve but worsen. She experienced uneasiness in swallowing water even with using a straw at the time of the first visit to our department.

During the physical examination, she exhibited severe mouth opening restriction with a maximum assisted mouth opening range of $10 \mathrm{~mm}$ with a hard end feel, but no temporomandibular joint (TMJ) pain on opening was noticed. No clinical findings associated with TMJ disk dysfunction, such as mandibular deflection and tenderness on both TMJs and masticatory muscles, were evident. She reported experiencing TMJ noise in the past.

Moreover, no clinical findings related to mucosal inflammation, swelling, pus discharge, or uncomfortable tooth exist. Interestingly, she manifested facial asymmetry due to involuntary contraction of the left facial expression muscles (Fig. 1).

The radiologic examination revealed the rarefying osteitis with osteosclerosis on the root of the mandibular left third molar, cortical erosion on the right condyle head, and shortening of the left condyle head (Fig. 2).

Considering the clinical findings of the simultaneous occurrence of acute trismus and dysphagia, rapid progression, facial muscle constriction, in addition to recent skin injury history, we strongly suspected that the patient has tetanus. Therefore, the patient was immediately referred to an infectious disease medicine specialist. After 2 weeks of admission treatment for tetanus in a general hospital, the patient recovered from tetanus and the trismus was resolved.

\section{DISCUSSION}

Trismus is a symptom commonly encountered by dental practitioners. The normal range of mouth opening in adults varies from 35 to $55 \mathrm{~mm}$ inter-incisally [6]. The diagnostic criteria for trismus vary among clinicians because no universal criteria for trismus were established. Some clinicians defined trismus as a mouth opening $<40 \mathrm{~mm}$, while others defined it as a mouth opening $<20 \mathrm{~mm}$ [7]. Thomas et al. [8] defined trismus according to a more gradual scale (light, above $30 \mathrm{~mm}$; moderate, above 15-30 mm; severe, below $15 \mathrm{~mm})$.

Trismus has several causes which range from the simple

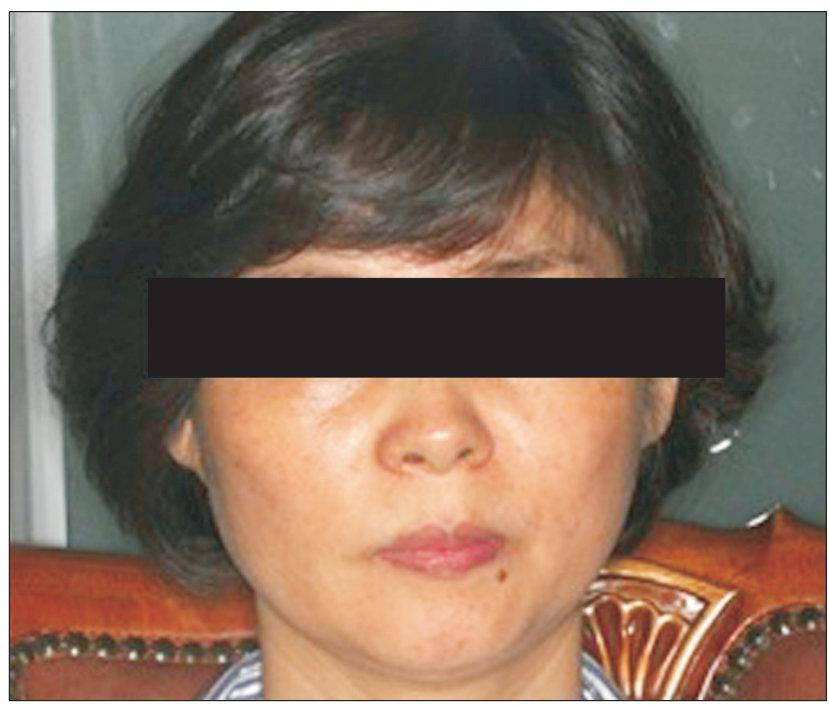

Fig. 1. The patient's left facial expression muscle contraction.
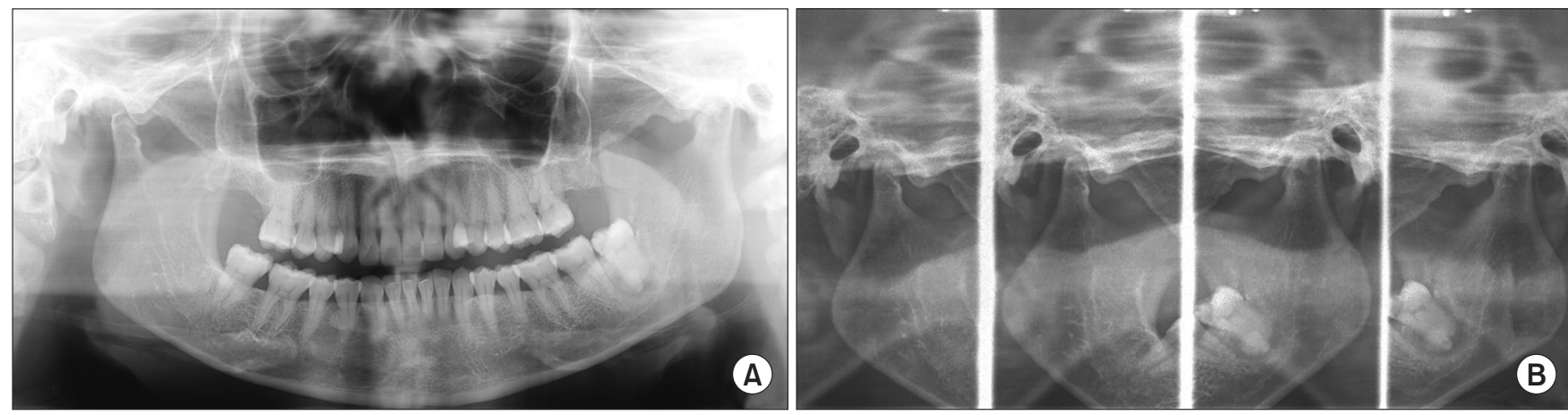

Fig. 2. Radiographic images. (A) Rarefying osteitis on the root of the mandibular left third molar in panoramic view. (B) Cortical erosion on the right condyle head and shortening of the left condyle head in temporomandibular joint panoramic view. 
and non-progressive to the potentially life-threatening. Several conditions may develop trismus including infection, trauma, dental treatment, TMJ disorders, tumors, drugs, radiotherapy, chemotherapy, congenital problems, and miscellaneous disorders [9]. Trismus can be divided into acute, subacute, and chronic depending on the type of development. Infection, trauma, postoperative conditions, drugs, malignant hyperthermia, and TMJ disease can be considered as causes of acute trismus [6].

In the case of acute trismus caused by maxillofacial infections (e.g., odontogenic infection, tonsillitis, pharyngitis, parotitis, and otitis), swelling and pain are seen on the affected side, and fever is common. In case of trauma, postoperative conditions, drugs, or malignant hyperthermia, the patient should be checked through careful history taking to rule out any possibility. Acute closed lock of the TMJ disc can occur due to displaced disc. In this case, the patient had a history of clicking sound and some TMJ discomfort. In closed lock conditions, the patient can open the jaw at about 25-30 mm, which is not severe trismus $[9,10]$. Myospasm is an involuntary tonic muscle contraction that causes acute trismus when affecting the masticatory muscles. In such a case, acute malocclusion is common, and pain occurs even at rest and increases with function. The affected muscle is firm and painful on palpation [10]. Usually, it occurs locally and appears unilaterally, but it is considered a systemic disease if multiple muscle spasm appears on both sides. Trismus has resulted from a variety of diseases, so it is important to make the correct diagnosis to effectively resolve the problem.

Tetanus is an acute infectious disease caused by a potent neurotoxin produced by $C$. tetani. The incubation period is usually about 8 days and may be up to several months $[11,12]$. C. tetani secretes two toxins, tetanospasmin and tetanolysin, of which tetanospasmin mainly leads to the clinical symptoms of tetanus [4]. Tetanospasmin binds with a high affinity to the presynaptic membrane of the motor neuron at the neuromuscular junction and enters the motor neuron axon terminals through endocytosis. It is retrogradely transported to the soma in the spinal cord and then transcytosis into inhibitory interneurons, blocking the release of inhibitory neurotransmitters such as GABA and glycine to motor neurons $[13,14]$. Consequently, the disinhibitory effect on the motor neuron results in the characteristic spasms of tetanus [4,15].

Generalized, localized, cephalic, and neonatal are the four main types of tetanus, depending on its clinical features, of which generalized tetanus is the most common type, accounting for approximately $80 \%$ of cases $[11,16]$. The early symptom of tetanus is trismus or lockjaw, followed by stiffness of the neck, difficulty in swallowing, and rigidity of the abdominal muscles as it progresses [11]. Among them, trunk stiffness can lead to opisthotonus and respiratory difficulty due to decreased chest wall compliance, which possibly leads to death [4].

The patient reported acute, severe trismus and dysphagia as chief complaints when visiting our department. Before coming to our department, she was prescribed antibiotics for 3 days because there seemed to be an odontogenic infection of the mandibular left third molar on the radiograph. However, the trismus was not resolved but worsened progressively thereafter. In this case, the acute closed lock of TMJ could be excluded from the clinical findings, showing simultaneous dysphagia and a more severe restriction range compared to the usual TMJ locking case. The patient showed multiple contractions of the facial and masticatory muscles, exhibiting a systemic disease such as multiple sclerosis and tetanus. The nature of the acute illness of this case rules out multiple sclerosis.

Tetanus is known as a vaccine-preventable disease with relative rarity in developed countries, but eradicating it is impossible because the causative agents are widespread in the environment $[4,15]$. According to the World Health Organization and Korea Centers for Disease Control and Prevention, approximately 30 cases still occur annually in South Korea $[17,18]$. However, because it is a low-prevalent disease, many clinicians have never actually experienced tetanus patients.

While tetanus is rare, it is a life-threatening disease when it occurs. The dentist is frequently the first medical person to encounter tetanic conditions because the main and earliest symptoms are trismus and dysphagia. However, due to the rarity of the disease, clinicians may be unfamiliar with the expressions the patient complains of and may not be suspicious of tetanus. No objective diagnostic test is available to confirm tetanus yet, and the diagnosis is entirely 
made by history and clinical findings [19]. It is hoped that the acute trismus diagnosis algorithm from Scully [6] will help clinicians make a prompt diagnosis when a patient presents with acute trismus. As dentists frequently encounter various trismus patients, it is important to understand the various causes of trismus, even if they are less likely to occur to avoid delayed diagnosis and poor prognosis. Therefore, the dentist should be familiar with the clinical characteristics of tetanus and should be able to refer to the appropriate medical clinician early in case of doubt.

\section{CONFLICT OF INTEREST}

No potential conflict of interest relevant to this article was reported.

\section{ORCID}

\author{
Seo-Hee Jang \\ https://orcid.org/0000-0002-0557-093X \\ Jin-Seok Byun \\ https://orcid.org/0000-0002-6182-1238 \\ Jae-Kwang Jung \\ https://orcid.org/0000-0003-3099-8097 \\ Jae-Kap Choi \\ https://orcid.org/0000-0001-6773-7507
}

\section{REFERENCES}

1. Schiavo G, Matteoli M, Montecucco C. Neurotoxins affecting neuroexocytosis. Physiol Rev 2000;80:717-766.

2. Masuyer G, Conrad J, Stenmark P. The structure of the tetanus toxin reveals pH-mediated domain dynamics. EMBO Rep 2017;18:1306-1317.

3. Giannini L, Maccari A, Chiesa V, Canevini MP. Trismus, the first symptom in a challenging diagnosis of tetanus. BMJ Case Rep 2016;2016:bcr2015213897.

4. Cook TM, Protheroe RT, Handel JM. Tetanus: a review of the literature. Br J Anaesth 2001;87:477-487.

5. Kyu HH, Mumford JE, Stanaway JD, et al. Mortality from tetanus between 1990 and 2015: findings from the global burden of disease study 2015. BMC Public Health 2017;17:179.

6. Scully C. Trismus. In: Scully C, ed. Oral and maxillofacial medicine: the basis of diagnosis and treatment. 3rd ed. St. Louis: Elsevier Health Sciences; 2013. pp. 167-169.

7. Dijkstra PU, Huisman PM, Roodenburg JL. Criteria for trismus in head and neck oncology. Int J Oral Maxillofac Surg 2006;35:337342.

8. Thomas F, Ozanne F, Mamelle G, Wibault P, Eschwege F. Radiotherapy alone for oropharyngeal carcinomas: the role of fraction size ( 2 Gy vs 2.5 Gy) on local control and early and late complications. Int J Radiat Oncol Biol Phys 1988;15:1097-1102.

9. Dhanrajani PJ, Jonaidel 0. Trismus: aetiology, differential diagnosis and treatment. Dent Update 2002;29:88-92, 94.

10. Okeson JP. Diagnosis of tempormandibular disorders. In: Okeson JP, ed. Management of temporomandibular disorders and occlusion. 8th ed. St. Louis: Elsevier Health Sciences; 2019. pp. 223258.

11. Centers for Disease Control and Prevention. Epidemiology and prevention of vaccine-preventable diseases. 8th ed. Washington, DC: Department of Health \& Human Services, Public Health Foundation; 2005.

12. Weng WC, Huang WY, Peng TI, et al. Clinical characteristics of adult tetanus in a Taiwan medical center. J Formos Med Assoc 2011;110:705-710.

13. Surana S, Tosolini AP, Meyer IFG, Fellows AD, Novoselov SS, Schiavo G. The travel diaries of tetanus and botulinum neurotoxins. Toxicon 2018;147:58-67.

14. Hassel B. Tetanus: pathophysiology, treatment, and the possibility of using botulinum toxin against tetanus-induced rigidity and spasms. Toxins (Basel) 2013;5:73-83.

15. Yen LM, Thwaites CL. Tetanus. Lancet 2019;393:1657-1668.

16. Pascual FB, McGinley EL, Zanardi LR, Cortese MM, Murphy TV. Tetanus surveillance--United States, 1998--2000. MMWR Surveill Summ 2003;52:1-8.

17. World Health Organization (WHO). Tetanus (total) reported cases [Internet]. Geneva: WHO; c2020. [cited 2020 Jul 15]. Available from: https://apps.who.int/immunization_monitoring/globalsummary/timeseries/tsincidencettetanus.html.

18. Korea Centers for Disease Control and Prevention (KCDC). Infectious disease portal [Internet]. Cheongju: Korea Centers for Disease Control and Prevention (KCDC); c2020 [cited 2020 Jul 11]. Available from: http://www.cdc.go.kr/npt/biz/npp/ist/simple/ simplePdStatsMain.do.

19. Pérez-Fernández J, Marín-García G, Rodríguez-Vega G. Tetanus. In: Hidalgo J, Woc-Colburn L, eds. Highly infectious diseases in critical care. Cham: Springer; 2017. pp. 203-212. 